\title{
Measurement of the Horizon Elevation for Satellite Tracking Antennas Located in Urban and Metropolitan Areas Combining Geographic and Electromagnetic Sensors
}

\author{
Jesús Nieves-Chinchilla , Mercedes Farjas , Ramón Martínez
}

\begin{abstract}
A B S T R A C T
In urban and metropolitan areas presence of nearby obstacles and signal interference sources surrounding tracking antenna locations reduces satellite communication times. In low orbit missions these obstructions are even more relevant due to a few minutes per satellite pass. In this scenario a best estimation of the antenna elevation mask in the mission frequency band is proposed to test the antenna design for a given site by applying mission simulation software before the installation stage. If needed, to improve efficiency in satellite tracking operations, mitigation techniques will be proposed either in the redesign or relocation of the antenna.

This paper describes a methodology for deriving the horizon elevation diagram, which includes a measurement setup when different data capturing sensors are used: geographic and electromagnetic. Application results which have contributed in the selection of the antenna location can be seen in the case study of the new CubeSat-LEO tracking antenna at Cal Poly University.
\end{abstract}

\section{Introduction}

Emergence and increase in the number of LEO (Low Earth Orbit) nano-satellite projects such as the QB50 project [1] and their development at universities and research centers in the last years, have conditioned the geo-location of the satellite tracking antennas in urban and metropolitan areas. Despite the great advantage of LEO orbits being the short distance the radio signal must pass through which in turn reduces transmission power requirements and minimizes the propagation delay [2], it is becoming necessary to increase the operational quality of the antenna. Critical points identified are: visibility times of a few minutes in each satellite pass per day, assembly adaptability to any direction in azimuth and elevation, accuracy of the initial orientation derived from the position of the satellite and the antenna, and effectiveness of satellite tracking through a continuous system in order to avoid possible signal losses. To improve the performance of the antenna design there are several solutions: the use of azimuth-elevation pedestals to minimize tracking losses [3], the antenna size selection to achieve the desired gain margin [4], and the antenna positioning system using stepper motors [5]. Moreover, in urban and metropolitan areas obstructions caused by buildings and interference sources become another critical point as they reduce the FOV (Field of View) and disturb the signal communication between the satellite and the antenna. In particular AOS (Acquisition of Signal) and LOS (Loss of Signal) along the horizon in certain azimuth directions, since above mentioned critical points in the tracking antenna operations increase. In this scenario for LEO tracking antennas, mission analysts must take into account the quality link and the mission concept when analyzing the link budget. Link budget analysis takes into account in situ analysis of external noise caused by radio electric emissions from the earth and the atmosphere, extraterrestrial sources, and man-made noise [6]. In this sense, measurements presented by Leferink et al. [7] confirm that different interference sources coming from industrial production plants, cars and trains affect radio links in UHF (Ultra High Frequency) and in VHF (Very High Frequency) bands. For LEO tracking antennas locations on building roofs, parameters such as the antenna gain in relation to its size and the noise temperature at in relation to spectrum congested antenna sites [8] are of great relevance in the link budget analysis in order to establish a robust communication link. From the link quality point of view, applying a standard antenna elevation mask with a fixed minimum elevation of $10^{\circ}$ would guarantee adequate conditions for the satellite 
communication links, as this would avoid most of the surrounding obstacles and interferences. [9]. However, from the mission concept point of view the above mentioned standard mask reduces the amount of data download per satellite pass in a science mission. This is further reduced as the altitude of the satellite decreases, the opportunities to track or communicate with it from the antenna become more restricted further complicating satellite scheduling [10]. In this scenario, a best estimation of the antenna elevation mask would increase the satellite FOV (field of view) and in consequence would maximize science data download. In this sense, results presented by Gill et al. [11] do not consider the satellite FOV as a link budget parameter but for the amount of telemetry data in downlinks with minimum possible elevations.

This paper analyzes the tracking antenna scenario associated to a new generation of nanosatellites called CubeSat [12]. These small satellites reduce the link budget margin available in LEO missions, adding short lifetime and limitation of available signal power on board as specific link budget parameters. In order to reach an optimal solution, considering the above mentioned points of view, a balance between them and the limited resources in CubeSat missions, a methodology to simulate the horizon elevation from the selected antenna site is proposed.

\section{Methodology}

This methodology has been developed taking into account the data set required by the satellite tracking software from the antenna location point of view. From the measurement data analysis, a measurement setup has been designed to finally select the most adequate equipment for data capturing processes.

\subsection{Measurement data}

The tracking software with information of the satellite orbit is engaged to the antenna rotor controller to start the tracking process when the satellite is in view (satellite entry) over a predefined elevation. The rotor controller governs the operation of the antenna rotors to generate the required voltages to steer the antenna in the correct angular position. Then, the antenna comes to stow position once the satellite goes below the minimum elevation (satellite exit). These programs, such as Orbitron [13] used to predict when and in which direction the satellite is available to communicate with it, or STK (Satellite Tool Kit) [14] used to analyze the satellite missions, require a data set from the antenna location point of view; first, to simulate and analyze the satellite mission, and; second, to control the positioner to point the antenna in the satellite direction during the tracking mission. [15]. The data set contains both the geographic coordinates and the orthometric height of the antenna rotor center, and the antenna elevation mask or altimetry profile surrounding the antenna site. In urban and metropolitan areas, this mask should also contain information about antenna minimum elevations to avoid physical and electromagnetic obstructions caused by nearby obstacles and signal interference sources which affect the communication links in certain azimuth directions from the antenna location site. Fig. 1 shows the simulation of this scenario in which nearby obstacles (white dots) surrounding the antenna site reduce the satellite FOV, and a radio amateur antenna that emits in the same frequency band also reduce the communication time between the satellite and the antenna in the azimuth direction of the satellite entry.

This required measurement data is twofold: geographic and electromagnetic. Considering the STK file required to create the scenario the data must be exported with the same parameters (azimuth, elevation) from each software used. In addition, the electromagnetic and geographic data fusion requires data capture in the same coordinate reference system.

\subsection{Measurement setup}

Considering that the purpose is to in situ simulate the tracking antenna elevation mask and the measurement data required, a measurement setup has been designed when using different data capturing sensors to provide in situ quick results by a swift process of data fusion. Data capturing sensors for each procedure have been selected for each procedure analyzing the data set required. Fig. 2 shows the scheme of the measurement setup designed which contains the procedures followed to obtain each mask, geographic and electromagnetic. In the first procedure, the Geographic study, the main purpose is to establish the spatial position of the antenna rotor center as the center of the coordinate reference system by using GNSS (Global Navigation Satellite System) technology. This reference system will be used for the acquisition of the altimetry profile data by using topographic instrument. The reference system will also be used in the second procedure, the Electromagnetic

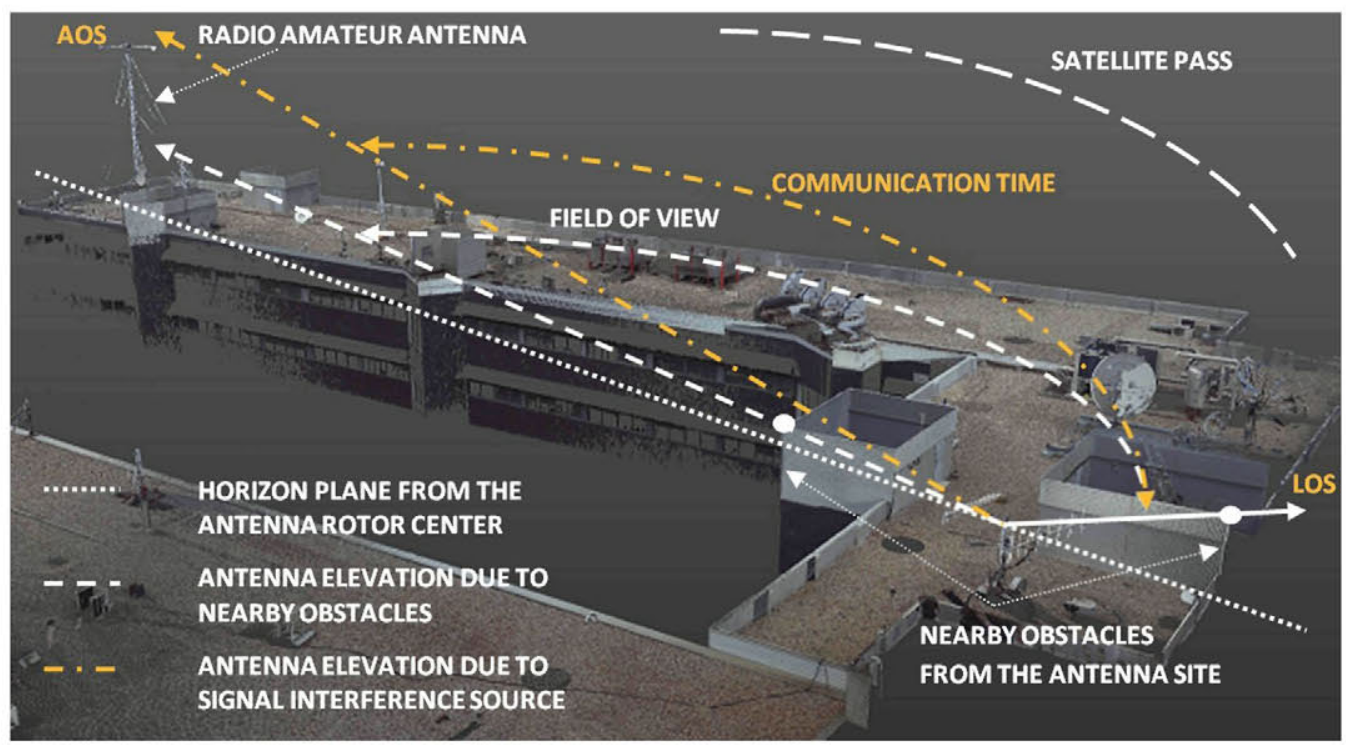

Fig. 1. Simulation of a tracking antenna scenario in a particular satellite pass. 


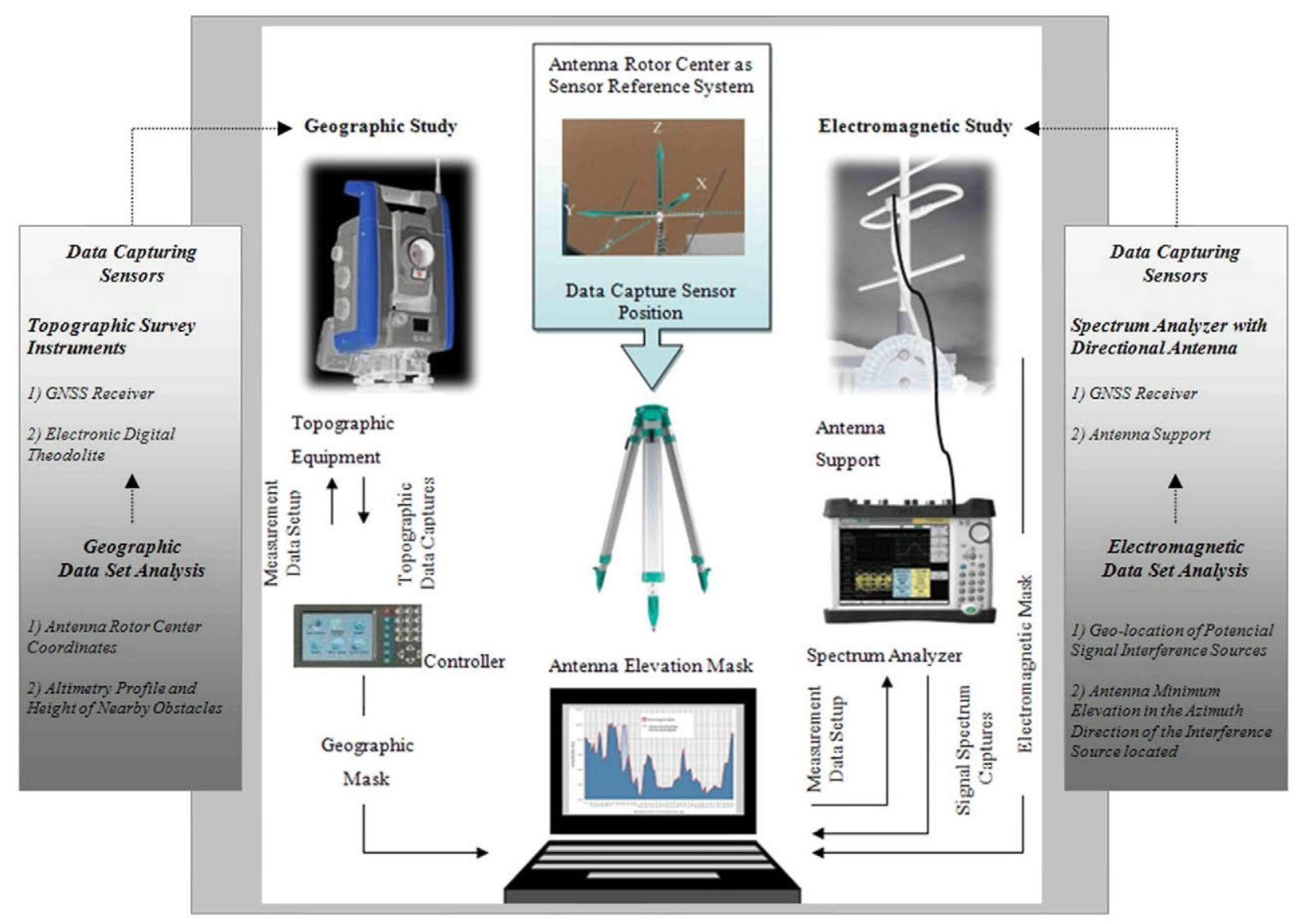

Fig. 2. Scheme of the designed measurement setup by applying the data set analysis and the adequate selection of the data capturing sensors in each procedure.

study, for the acquisition of signal transmission data from the interference sources by using spectrum analyzer equipment.

The hardware setup designed takes into account the use of different data capturing sensors. An antenna support was designed for the electromagnetic study to establish the same reference system for the data acquisition process as in the geographic study. This support was designed considering how a theodolite works, replacing the viewfinder by a cylinder to insert the directional antenna. This cylinder is contained in the vertical plane and includes an inclinometer which allows targeting elevations during the data acquisition by using a spectrum analyzer in a specific azimuth direction. In addition, the designed measurement setup has been adapted so it can be used for higher antennas: first, by replacing the tripod for an extendable mast; second, by using a topographic instrument with digital camera integrated, and; third, establishing a wireless connection for both data links, input and output.

The core of the measurement setup is the software configuration both in input and output data before the data acquisition processes. In particular, the output data setup in the parameters required to simulate the antenna horizon elevation (azimuth, elevation), provides a quick data fusion process by a basic calculation program. In the geographic study, selecting output data in angular coordinates by the portable controller, and; in the electromagnetic study by the antenna support oriented in a specific azimuth direction, and targeting the antenna minimum elevation by the inclinometer integrated for a specific signal threshold. Further description of both procedures and result by applying the data fusion process will be describe in the experimental setup applied in the case study at Cal Poly University.

\section{Case study at Cal Poly University}

The proposed measurement setup has been experimented in the future installation site of the Cal Poly's third antenna. The antenna will be able to track CubeSat-LEO in VHF and UHF bands and it will be capable of achieving significantly higher data rates than Cal Poly's two other antennas, Marconi and Hertz [16].

\subsection{Geographic study}

The main purpose in the study application is the antenna selection site within a location area by comparing the available visibility times between site proposals. In this case study, for a given antenna site, the first step was the analysis of the most adequate data acquisition process to update the geographic information surrounding the antenna location. By applying the geographic data fusion of the altimetry profile and information of nearby obstacles a best estimation of the satellite FOV is obtained considering the satellite FOV loss when applying a $10^{\circ}$ elevation mask (see Fig. 3 (a)). To establish the accuracy of the required measurement data the standard antenna rotation is $0.5^{\circ}$ and an antenna rotor center $2.20 \mathrm{~m}$ high was used. The second step was to establish this antenna point as the center of coordinate reference system (see Fig. 3(b)) by capturing its spatial position and orthometric height using GNSS technology. The OPUS (Online Positioning User Service) system [17], which provides coordinates in the NSRS (National Space Reference System), was used. The third step was the capture of the altimetry profile (azimuth, elevation) from this position, once the measurement data setup of the topographic controller 


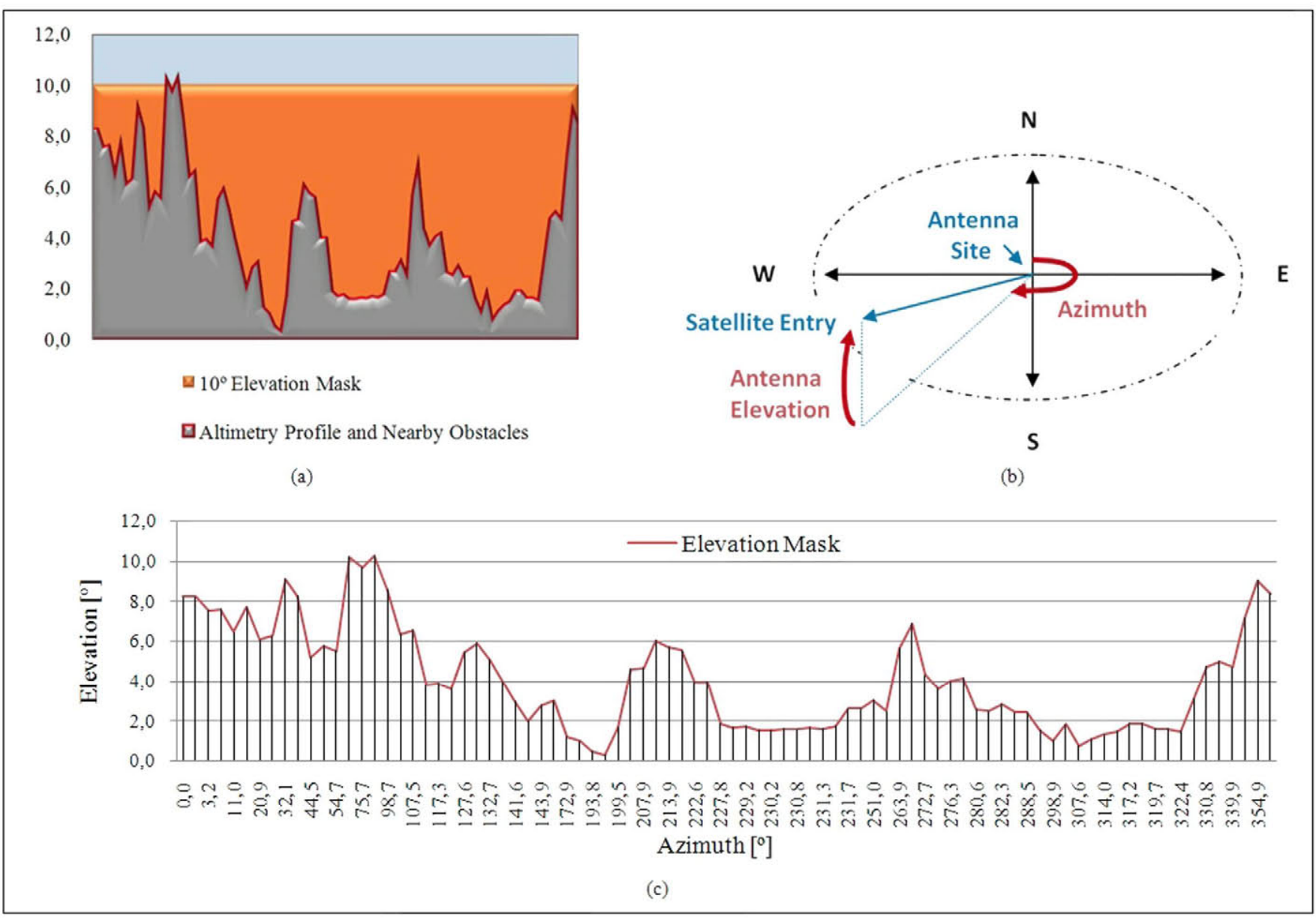

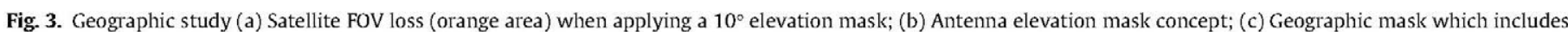

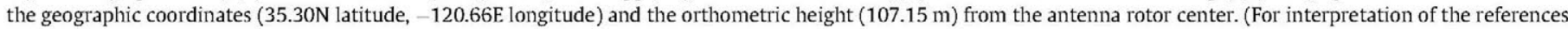
to color in this figure legend, the reader is referred to the web version of this article.)

was done. Fig. 3(c)) shows the graphic result which contains antenna minimum elevations along the horizon visible from the antenna site. This data set (azimuth, elevation) and the spatial position of the antenna rotor center compose the geographic mask, which is required by the satellite tracking programs to simulate the mission, from the point of view of the visibility times.

\subsection{Electromagnetic study with the directional antenna}

The main purpose in the study application is completing the geographic mask information from the point of view of the interfering signals surrounding the antenna site. The presence of signal interference sources which emit in the mission frequency bands, reduce the available visibility times along the horizon from the antenna site to establish satellite communication links. Within a selected location area to install the antenna, an omnidirectional antenna can be used for a preliminary detection of interference sources surrounding the site proposals, and by seeking the strongest signal in the mission frequency bands can be very useful for the antenna site selection with low interfering signals. In this case study, for a given antenna site, the purpose was then the electromagnetic study with the directional antenna to complete the antenna elevation mask obtained by applying the geographic study. The first step was the geo-location of the interference sources in the frequency ranges of the mission bands by targeting the azimuth directions from the antenna site. The second step was the simulation of the antenna spatial position in the azimuth range affected by a specific interference source, by targeting the antenna minimum elevation in order to establish a satellite radio link under a predefined signal threshold.

\subsubsection{Geo-location of signal interference sources}

The process proposed in the methodology consists in the geolocation of signal interference sources by following three stages; detection; identification and; location [18]. In this scope, the most efficient solution proposed is to analyze mapped signals from export field strength measurements obtained using GNNS technology based location information, and transfer it to PC-based mapping programs [19] which can be then displayed by the Google Earth application. Due to equipment available at the University, spectrum analyzer equipment without integrated GNSS technology, the proposed geo-location solution was achieved by combining visual inspection and information given at the facility manager's office. Once the main interference sources were identified, their azimuth directions from the antenna site were targeted using topographic equipment. Fig. 4 shows the azimuth directions of the interferences sources identified.

Following the proposed procedure, the next steps were to setup both hardware and software for the electromagnetic study. First, adjustment of the spatial position of the antenna rotor center in the antenna support applying the same coordinate reference system as in the geographic study was performed. Second, the frequency band ranges assigned by International Telecommunication Union-Recommendations (ITU-R) in CubeSat mission projects 


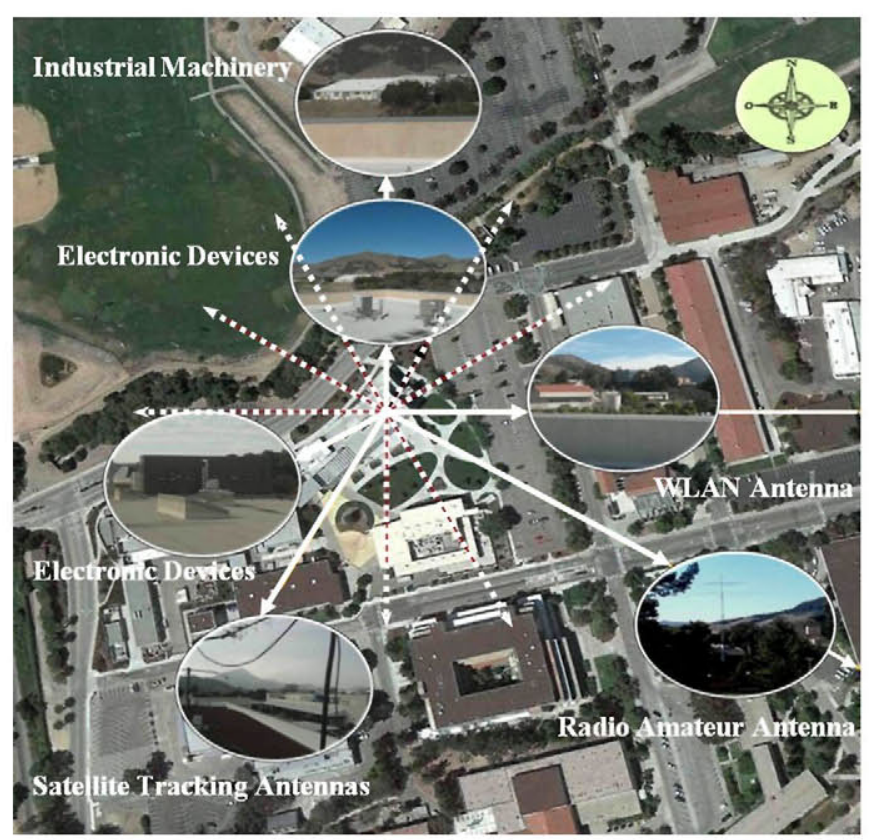

Fig. 4. Top view (source: Google Earth) of the building geo-location which includes the azimuth directions of the signal interference sources identified; $0^{\circ}$, air conditioning devices and industrial machinery; $90^{\circ}$, wireless mobile antenna; $120^{\circ}$, radio amateur antenna; $210^{\circ}$, satellite tracking antennas; $240^{\circ}$, building climate devic.

during the last 10 years in UHF and in VHF band [20] were selected. Finally, the measurement parameters as applied were: a minimum sweep time of $300 \mathrm{~s}$, a frequency span of $3.000 \mathrm{MHz}$, a resolution bandwidth (RBW) of $30 \mathrm{kHz}$, an input attenuation of $10 \mathrm{kHz}$ and, and a video bandwidth (VBW) of $10 \mathrm{kHz}$ [21]. In addition, to verify presence of other signal sources during the data capturing process this was completed by analyzing signal transmissions, each $30^{\circ}$ along the horizon (see Fig. 4). No relevant results were obtained.

Fig. 5(a) shows the designed hardware setup considering the spatial position established in the geographic study thanks to the use of the designed antenna support and an extendable tripod. To describe next step, the Electromagnetic mask, a radio amateur antenna located in the $120^{\circ}$ azimuth direction, which emits in VHF band, was selected as a particular case of a signal interference

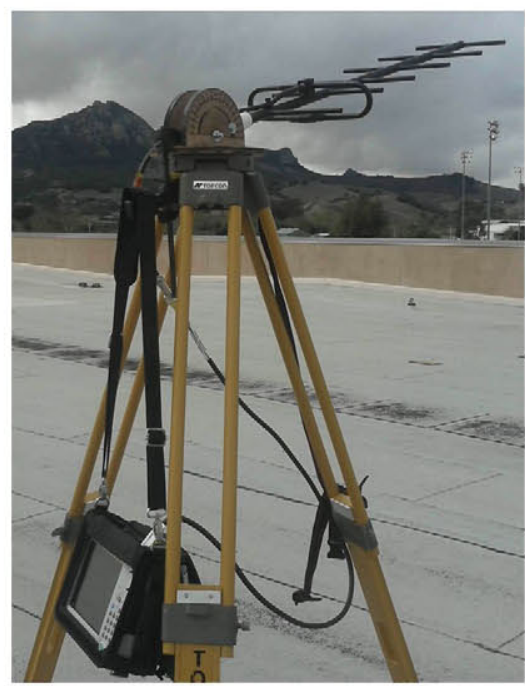

Fig. 5. Hardware setup for the electromagnetic study with the spectrum analyzer equipment with the directional antenna. source which affects the AOS in this specific azimuth direction (see Fig. 6).

\subsubsection{Electromagnetic mask}

The purpose of the measurement setup is to capture the electromagnetic mask by the simulation of the antenna spatial position in the azimuth range affected by a specific interference source, and to target the minimum antenna elevation to establish a satellite radio link under a predefined signal threshold. Results evidences how the signal power transmission decreases as the antenna elevation is increased from $0^{\circ}$ to $20^{\circ}$ (see Fig. 7), considering a threshold of $80 \mathrm{dBm}$ as the standard CubeSat power transmission, and a signal power range of $10-15 \mathrm{dBm}$ to mitigate the disturbance. An antenna elevation of $20^{\circ}$ was established for a clear communication link with the satellite in this specific azimuth direction (see Fig. $7(\mathrm{~d})$ ). The proposed measurement setup can be applied to any minimum elevation threshold between 5 and 20 degrees as used in real missions.

The azimuth range which is affected by the signal interference beam should be considered to complete the electromagnetic mask information. Taking into account the azimuth direction of the interference source as the central axis of the affected area, the next step was the analysis of the signal power received in $90^{\circ}$ and $150^{\circ}$ azimuth directions. Table 1 shows peak power results of the signal received, applying the same measurement system as in the azimuth direction of the radio amateur antenna to determine the signal power profile in the azimuth range affected by the interference source.

Fig. 8 shows the area of the geographic mask, including the main elevations from the antenna site, which is affected in the VHF band by the radio amateur antenna located in the $120^{\circ}$ azimuth direction. Curves in Fig. 9, represent the signal peak power received between $90^{\circ}$ to $150^{\circ}$ azimuth directions in specific antenna elevations $\left(0^{\circ}, 10^{\circ}\right.$ and $\left.20^{\circ}\right)$ and, how the signal power decreases as the antenna turns away from the center of the source signal beam source. The resulting area represents the electromagnetic mask which completes the geographic information in this azimuth range, and allows establishing the antenna minimum elevation to start communication with the satellite in the VHF band [22,23].

Electromagnetic measurements in other frequency bands require the adaptation of the measurement setup. For instance, interference detection in the $2 \mathrm{GHz}$ band with the presence of

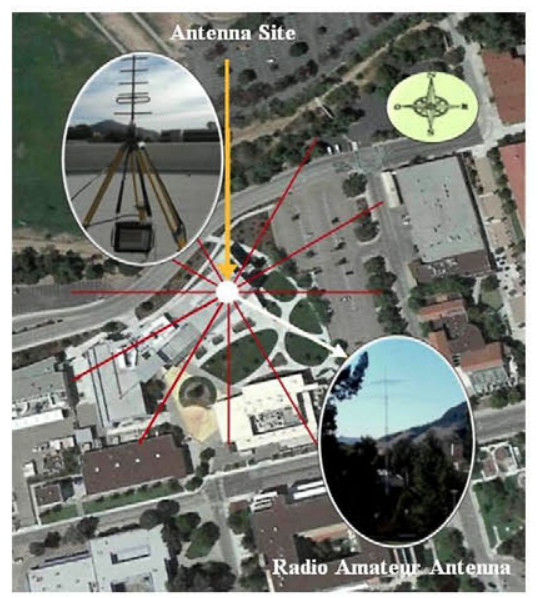

Fig. 6. Top view (source: Google Earth) of the building geo-location which includes the antenna site (orange line) and the azimuth direction (white line) of the radio amateur antenna from the antenna spatial position. (For interpretation of the references to color in this figure legend, the reader is referred to the web version of this article. 


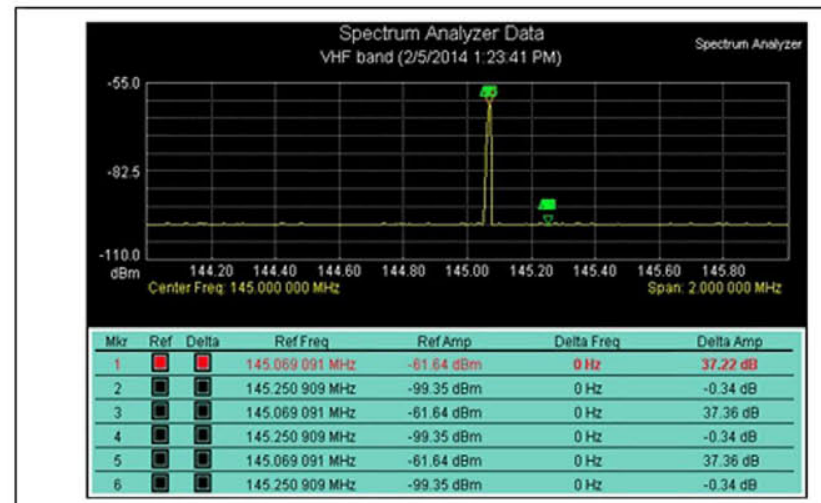

(a)

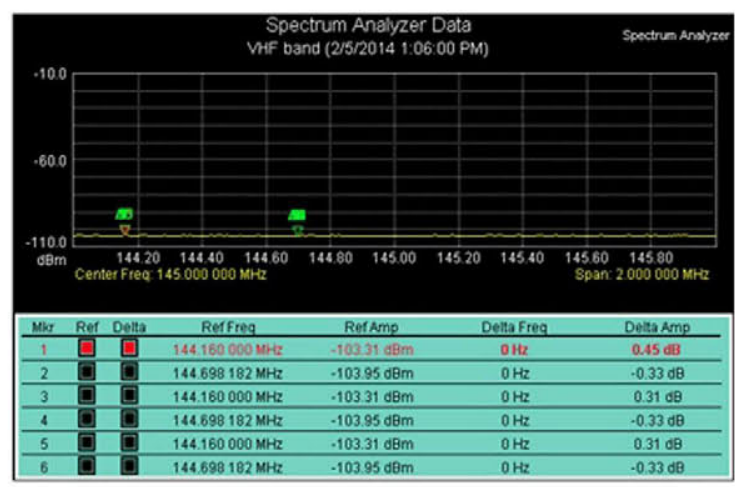

(c)

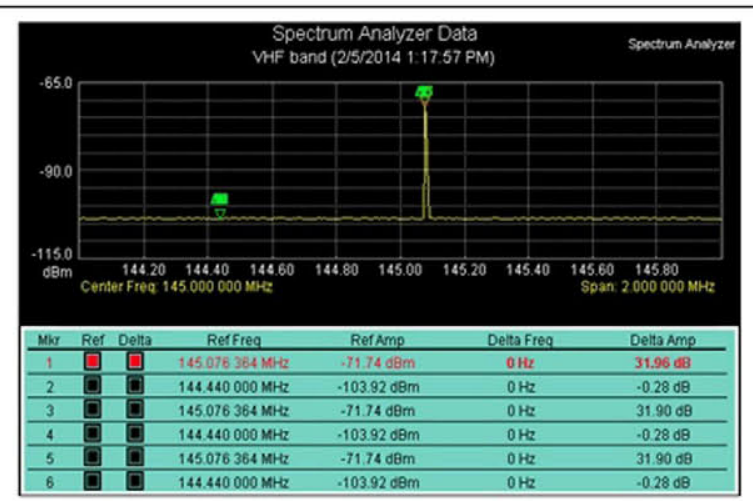

(b)

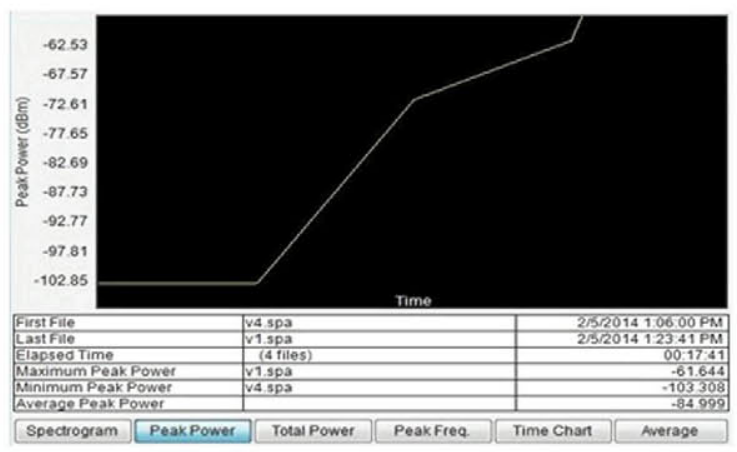

(d)

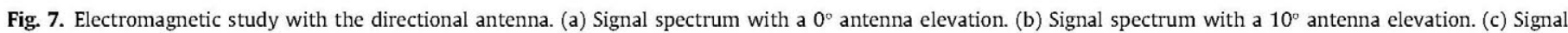
spectrum with a $20^{\circ}$ antenna elevation. (d) Graphic representation of the measurement data by the signal power function.

Table 1

Results of the signal peak power $(\mathrm{dBm})$ received in the azimuth range established.

\begin{tabular}{|c|c|c|c|c|}
\hline \multirow{4}{*}{ 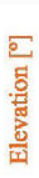 } & 20 & $-103,48$ & $-103,31$ & $-103,6$ \\
\hline & 10 & $-98,92$ & $-71,74$ & $-103,62$ \\
\hline & 0 & $-103,48$ & $-61,64$ & $-103,6$ \\
\hline & & 90 & 120 & 150 \\
\hline \multicolumn{5}{|c|}{ Azimuth $\left[^{\circ}\right]$} \\
\hline
\end{tabular}

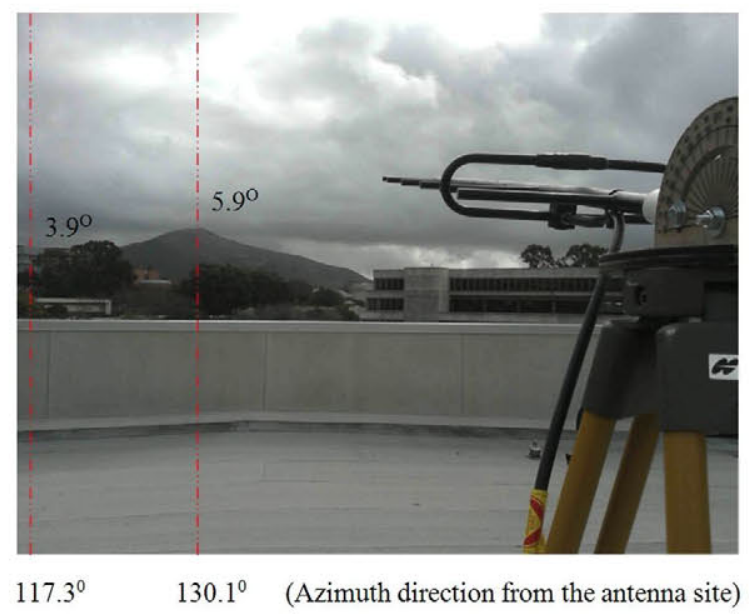

Fig. 8. Measurement setup applied in the azimuth range affected where the radio amateur antenna was located.
802.11 and/or 802.15 .4 networks $(2.4-2.4835 \mathrm{GHz})$ requires the filtering of the particular frequency channel used by the satellite downlink to avoid confusion between the interference in the frequency channel with interference in a neighbor frequency channel. Thus, appropriate bandwidth and central frequency parameters in the spectrum analyzer must be selected.

\subsection{Fusion of the geographic and electromagnetic masks}

Fusion of both masks, geographic and electromagnetic, gives the expected result for the in situ simulation of the tracking

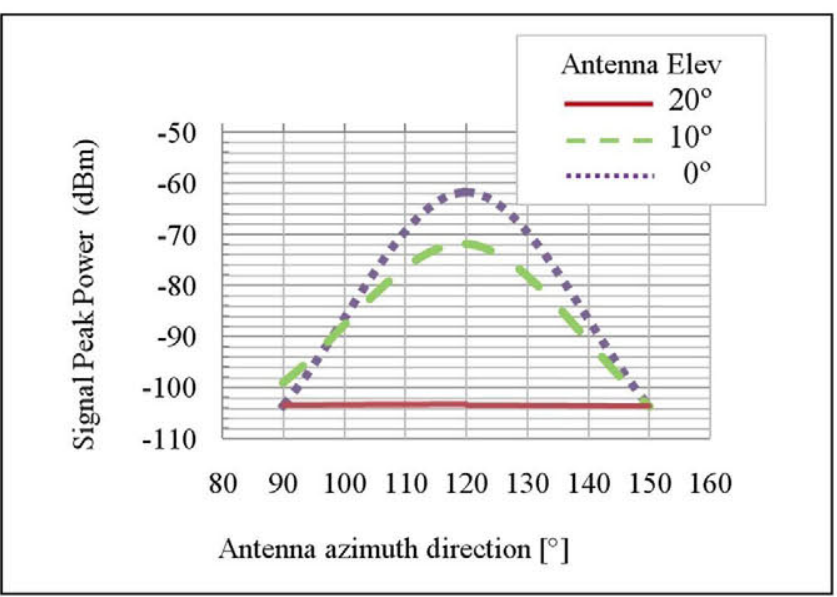

Fig. 9. Graphic representation of the measurement data captured from the spectrum analyzer with the directional antenna in the VHF band. 


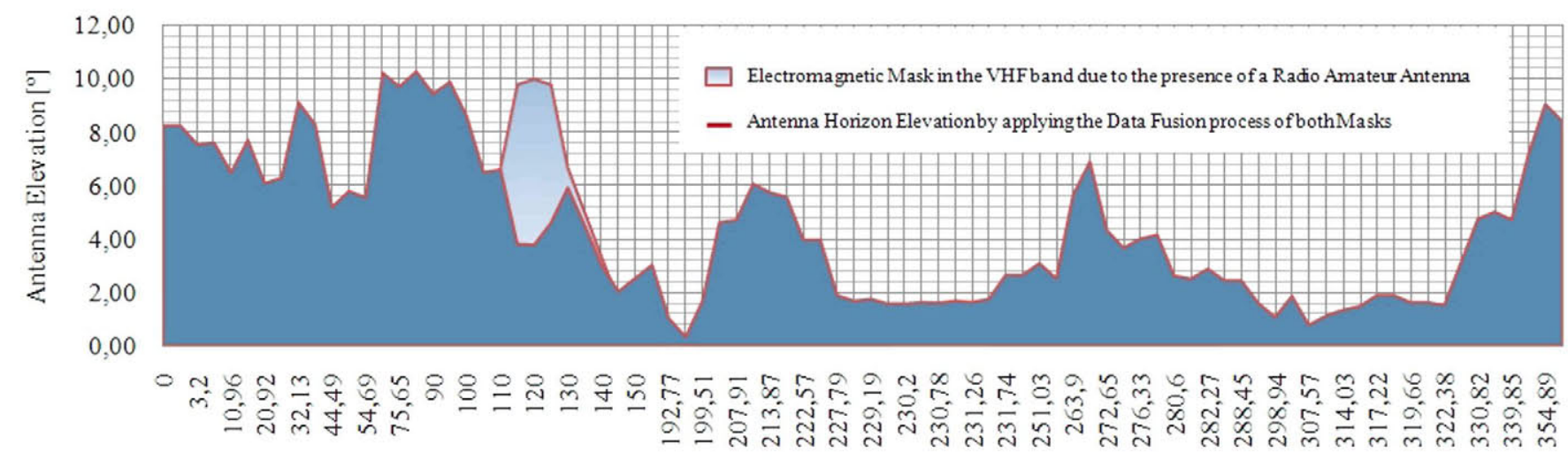

Azimuth direction from the Antenna site selected [ $\left.{ }^{\circ}\right]$

Fig. 10. Graphic representation of the tracking antenna elevation mask customized in the VHF band, from the future location site at Cal Poly University.

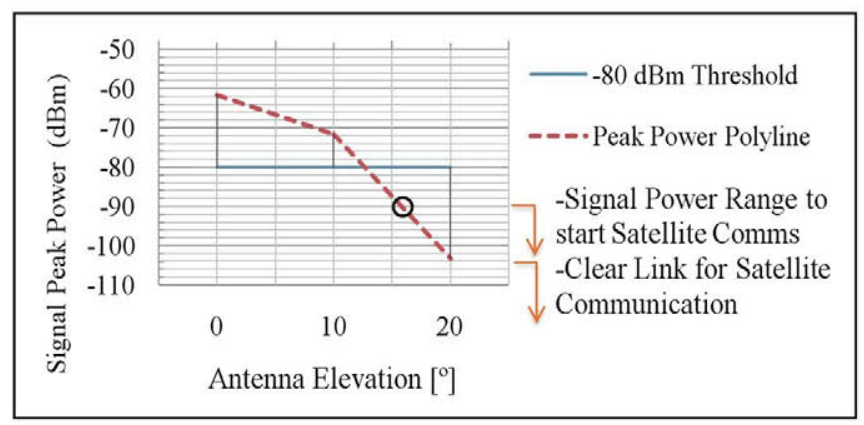

Fig. 11. Graphic representation of the further analysis proposed for a best estimation of the antenna elevation to start communications in a $10-20^{\circ}$ elevation range.

antenna elevation mask in the VHF band from the selected site. The swift data fusion process including application of a basic calculation program was possible through the hardware setup by establishing the same reference system when using different capturing sensors and, through software setup by transforming the output data in the parameters required, azimuth and elevation, in both studies. Fig. 10 shows the graphic representation of the antenna elevation customized mask in the VHF band from the proposed site. This mask combines the geographic mask information (see Fig. 3(c)) and the electromagnetic mask information, captured in the azimuth range affected by radio amateur antenna (see Table 1).

\section{Conclusions and future works}

As initially stated, a standard antenna elevation mask would typically guarantee adequate conditions to establish a satellite radio link in urban and metropolitan areas, as this would avoid most of the surrounding obstacles and interferences. However, obstructions which cannot be avoided often hinder such a standard mask from ensuring an optimal satellite radio link. In this sense, this paper concludes that a customized mask which takes into account the updated geographic and electromagnetic information surrounding the antenna site optimizes the satellite communication times which are available.

In the analyzed case study for CubeSat-LEO missions, the conclusion drawn is the usefulness of simulating the horizon elevation for a given site before antenna installation to test the antenna design. This provided relevant information for the implementation of mitigation techniques either in the redesign or relocation of the antenna to maximize the amount of telemetry data in downlinks as each of them would have the minimum possible antenna elevation.

The conclusion drawn from the experimented measurement setup is the swift data fusion process using geographic and electromagnetic data capturing sensors. This is the result of previously configuring the software and hardware setup. In particular, the design of a directional antenna support in the hardware setup which allows the simulation of the antenna spatial position, azimuth and elevation, in the electromagnetic study.

Finally, there are several follow on issues that lead towards a generic method to measure the horizon elevation for satellite tracking antennas located in urban and metropolitan areas. These can be inferred from the proposed measurement setup. First, the implementation in the processing software for the electromagnetic mask of others link budget parameters relative to the earth station and satellite performance. Second, experimentation in other frequency bands which requires the adaptation of the proposed measurement setup. Finally, a further analysis of the electromagnetic mask, using a mechanical solution to calibrate the antenna support which includes the inclinometer. Fig. 11 shows why this improvement in the antenna support would determine a best estimation of the antenna minimum elevation to establish a radio link between an earth station and a satellite.

\section{Conflict of interest}

The authors declare no conflict of interest.

\section{Acknowledgements}

The authors would like to acknowledge to Professor Jordi PuigSuari at the Cal Poly University for the opportunity given to Jesús Nieves-Chinchilla to apply his doctoral research, and also to Dr. Ricardo Tubio-Pardavila and to the PolySat team, in particular to Jimmy Tang.

\section{References}

[1] QB50, An FP7 project, <https://qb50.eu/index.php/project-description-obj>.

[2] R. Elbert, Introduction to Satellite Communication, $2^{n}$ ed., Artech House, Norwood, Massachusetts, UK, 1999, p. 23.

[3] K. Willey, Selecting a Pedestal for tracking LEO Satellites at Ka Band, Microwave J. (2000), April $1<\mathrm{http}: / /$ www.microwavejournal.com/articles/ 2937-selecting-a-pedestal-for-tracking-leo-satellites-at-ka-band>.

[4] K. Willey, Antenna selection to minimize pointing requirements, Microwave J. (2002), January 1 <http://www.microwavejournal.com/articles/3371-antennaselection-to-minimize-pointing-requirements>. 
[5] Emad A. Gabbar, Moh. Alhasan, abdelrasoul jabar alzubaidi, A design software driver for a satellite dish antenna positioning system, IOSR J. Eng. 5 (2015) 42 44. <http://www.iosrjen.org/Papers/vol5_issue1\%20(part-2)/F05124244.pdf>.

[6] G. Vasilescu, External Noise, in: Electronic Noise and Interfering Signals: Principles and Applications, Springer, Berlin, Heidelberg, Germany, 2005, pp. $255-257$.

[7] F. Leferink, F. Silva, J. Catrysse, S. Batterman, V. Beauvois, A. Roc'h, Man-made noise in our living environments, Radio Sci. Bull. 334 (2010) 49-57. <http:// purl.utwente.nl/publications/75120>.

[8] R. Elbert, Satellite Links, Multiple Access Methods, and Frequency Bands, in: The Satellite Communication Applications Handbook, second ed., Artech House, Boston; London, UK, 2004, pp. 28-46.

[9] Sh. Cakaj, W. Keim, K. Malaric, Communication duration with low earth orbiting satellites, in: Proceedings of the 4th International Conference on Antennas, Radar and Wave Propagation, Montreal, Québec, Canada, 2007.

[10] D. Vallado, W. McClain, Mission analysis, in: Fundamentals of Astrodynamics and Applications, 2nd ed., Microcoms Press, El Segundo, CA, USA, Kluwer Academic Publishers, Dordrecht, The Netherlands, 2001, p. 829.

[11] E.K.A. Gill, C. Verhoeven, K. Gill, M. De Milliano, A New Approach for Enhanced Communication to LEO Satellites, American Institute of Aeronautics and Astronautics (AIAA), 2010.

[12] H. Heidt, J. Puig-Suari, A. Moore, S. Nakasuka and R. Twiggs, CubeSat: A new Generation of Picosatellite for Education and Industry Low-Cost Space
Experimentation, in: Proceedings of the 13th Annual AIAA/USU Small Satellite Conference, Logan, UT, USA, 2000.

[13] Orbitron-Satellite Tracking System, <http://www.stoff.pl>.

[14] System Toolkit, AGI Inc., <http://www.agi.com/products/stk>

[15] R. Martínez, S.R. Diaz, F.R. Vedal, Educational ground station based on software defined radio, in: Proceedings of the 59th International Astronautical Congress, Glasgow, UK, 2008.

[16] PolySat, <http://polysat.calpoly.edu>.

[17] OPUS, <http://www.ngs.noaa.gov/OPUS>.

[18] JDSU, <http://www.jdsu.com/Productliterature/Interference an_nsd tm_ae pdf>.

[19] Anritsu, <http:/downloadfile.anritsu.com/RefFiles/en-US/Products-Solutions/ RF-Interference-Hunting-Techniques.pdf>.

[20] Papers and Presentations by Bryan Klofas, <http://www.klofas.com/papers/ Klofas_Communications_Survey_2009-2012.pdf>.

[21] Anritsu, <http://www.anritsu.com/en-US/Products-Solutions/Products/MST aspx>.

[22] J. Nieves-Chinchilla, M. Farjas, R. Martínez, Analysis methodology for optimal selection of ground station antennas site in space missions, in: Proceedings of the American Geophysical Union's 46th Annual Fall Meeting, San Francisco, CA, USA, 2013.

[23] J. Nieves-Chinchilla, M. Farjas, R. Martínez, Optimization of the antenna elevation mask in CubeSat missions., in: Proceedings of the 11th Annual CubeSat Developers' Conference, San Luis Obispo, CA, USA, 2014. 